\title{
Coding metamaterials, digital metamaterials and programmable metamaterials
}

\author{
Tie Jun Cui ${ }^{1,2, *}$, Mei Qing Qi ${ }^{1, *}$, Xiang Wan ${ }^{1, *}$, Jie Zhao ${ }^{1}$ and Qiang Cheng ${ }^{1,2}$
}

Metamaterials are artificial structures that are usually described by effective medium parameters on the macroscopic scale, and these metamaterials are referred to as 'analog metamaterials'. Here, we propose 'digital metamaterials' through two steps. First, we present 'coding metamaterials' that are composed of only two types of unit cells, with 0 and $\pi$ phase responses, which we name ' 0 ' and ' 1 ' elements, respectively. By coding ' 0 ' and ' 1 ' elements with controlled sequences (i.e., 1-bit coding), we can manipulate electromagnetic (EM) waves and realize different functionalities. The concept of coding metamaterials can be extended from 1-bit coding to 2-bit coding or higher. In 2-bit coding, four types of unit cells, with phase responses of $0, \pi / 2, \pi$, and $3 \pi / 2$, are required to mimic the '00', '01', ' 10 ' and ' 11 ' elements, respectively. The 2-bit coding has greater freedom than 1-bit coding for controlling EM waves. Second, we propose a unique metamaterial particle that has either a ' 0 ' or ' 1 ' response controlled by a biased diode. Based on this particle, we present 'digital metamaterials' with unit cells that possess either a '0' or ' 1 ' state. Using a field-programmable gate array, we realize digital control over the digital metamaterial. By programming different coding sequences, a single digital metamaterial has the ability to manipulate EM waves in different manners, thereby realizing 'programmable metamaterials'. The above concepts and physical phenomena are confirmed through numerical simulations and experiments using metasurfaces.

Light: Science \& Applications (2014) 3, e218; doi:10.1038/lsa.2014.99; published online 24 October 2014

Keywords: coding; digital; metamaterial; metasurface; programmable

\section{INTRODUCTION}

Metamaterials are artificial structures that are engineered to obtain unusual properties, ${ }^{1}$ such as negative refraction, ${ }^{2,3}$ perfect lensing or superlensing, ${ }^{4}$ and invisibility cloaking. ${ }^{5-11}$ The current metamaterials are generally classified into two types on the basis of their structural arrangements: homogeneous (with periodic structures) and inhomogeneous (with non-periodic structures). ${ }^{1}$ The primary studies on metamaterials focus on periodic structures with subwavelength scales, which are described by uniform macroscopic medium parameters. Using special designs of unit structures, electromagnetic (EM) waves can be controlled either by extreme values of effective permittivity and permeability (e.g., negative refraction and perfect lensing owing to a negative index of refraction ${ }^{2-4}$ and high-directive emission and tunneling effects owing to a zero index of refraction ${ }^{12-14}$ ) or by anisotropic effective medium properties. ${ }^{15-17}$ For the periodic structures that cannot be described by the effective medium theory, such as photonic crystals, the allowed and forbidden energy bands are used to manipulate EM waves. ${ }^{18,19}$ In contrast, special designs of non-periodic structures with subwavelength geometries that change over a gradient, which normally behave as macroscopic inhomogeneous metamaterials, have considerably more freedom to control EM fields. Transformation optics ${ }^{5,6}$ provides a powerful tool for designing ideal anisotropic and inhomogeneous metamaterials to arbitrarily control wave propagations, polarizations, and scattering properties to realize unusual physical phenomena and novel devices, including invisibility cloaks, ${ }^{5-11}$ concentrators, $^{20}$ optical illusions ${ }^{21,22}$ and novel lenses. ${ }^{23,24}$ The conventional geometrical optics method and Fermat principle provide an alternative approach for designing gradient refractive index metamaterials ${ }^{25}$ for generating high-performance antennas, ${ }^{26-28}$ high-resolution imaging lenses, ${ }^{29}$ low scattering cross-sections ${ }^{30}$ and even for performing mathematical operations. ${ }^{31}$ Recently, metasurfaces, either periodic or gradient metasurfaces, have also been investigated to manipulate EM waves. ${ }^{32-38}$

Based on the effective medium theory, existing metamaterials, either periodic or non-periodic structures, are usually characterized by continuously macroscopic media with effective permittivity and permeability, either homogeneous or inhomogeneous. The 'continuous' feature results in the existing metamaterials being classified as 'analog metamaterials'. Della Giovampaola and Engheta recently presented a method for constructing 'metamaterial bytes' through proper spatial mixtures of 'digital metamaterial bits', ${ }^{39}$ in which the 'digital metamaterial bits' are some material particles that possess distinct material properties (e.g., Si with a positive permittivity and Ag with a negative permittivity). However, the resulting metamaterial bytes are still described by the effective medium parameters. ${ }^{39}$ Here, we propose the general concepts of 'coding metamaterial', 'digital metamaterial' and 'programmable metamaterial'. Here, digital metamaterial means that a single metamaterial can be digitally controlled to obtain distinctly different functionalities. To realize the coding metamaterial,

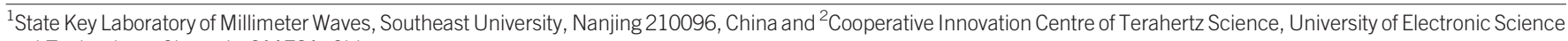
and Technology, Chengdu 611731, China

*These authors contributed equally to this work

Correspondence: Professor TJ Cui, State Key Laboratory of Millimeter Waves, Southeast University, Nanjing 210096, China

E-mail: tjcui@seu.edu.cn

Received 12 August 2014; revised 5 September 2014; accepted 9 September 2014 
we shall introduce two types of unit cells with 0 and $\pi$ phase responses to mimic the ' 0 ' and ' 1 ' elements for 1-bit digital such that they can be controlled using existing digital technology. These ' 0 ' and ' 1 ' elements do not need to be described by macroscopic medium parameters. By designing coding sequences of ' 0 ' and ' 1 ' elements in coding metamaterials, we can manipulate EM waves to obtain different functionalities. This concept can be extended to 2-bit or more.

In fact, the opposite-phase methods have been widely used in the antenna and optical communities. By designing a thin, artificial perfect magnetic conductor (with 0 phase) and combining the perfect magnetic conductor cells with perfect electric conductor (with $\pi$ phase) cells in a chessboard-like configuration, ${ }^{40}$ the reflections of any normally incident EM plane waves will cancel out, resulting in the reduction of radar cross-sections (RCSs). Similarly, the binaryphase (or multi-phase) grating has been intensively studied in diffractive optics, which are generated by multi-layered dielectric masks with designed etching patterns. ${ }^{41,42}$ For example, for a phase shift of $\pi$, the etching depth has to be approximately equal to the wavelength of light, ${ }^{41}$ which is electrically large for microwaves. The related area also includes spatial light modulators, ${ }^{43}$ which impose certain forms of spatially varying modulations on the light beams. By combining with metamaterial particles, metamaterial absorber spatial light modulators were realized in the terahertz frequencies. ${ }^{44-47}$

In this article, we propose coding metamaterials and demonstrate their ability to manipulate EM waves by using different coding sequences of ' 0 ' and ' 1 ' metamaterial particles. We further propose a unique metamaterial particle that can realize either a ' 0 ' or ' 1 ' element controlled by a biased diode. Then, we construct a digital metamaterial, which is composed of the unique particles with either the ' 0 ' or ' 1 ' state. By using field-programmable gate array (FPGA) hardware, we realize digital control over the digital metamaterial. Through numerical simulations and experiments, we demonstrate that a single digital metamaterial has distinct abilities for manipulating EM waves controlled by the FPGA program, thereby realizing a 'programmable metamaterial'.

\section{MATERIALS AND METHODS}

\section{1-bit coding metamaterials}

All results in this article are based on the concept of coding metamaterials. We start with 1-bit coding metamaterials. As shown in Figure 1a, we consider a special metasurface that is composed of binary digital elements of ' 0 ' or ' 1 '. The physical realization of digital elements is not unique, but it requires distinct responses to obtain significant phase changes in order to have considerable freedom to control EM waves. In the binary case, the maximum phase difference is $\pi$ (or $180^{\circ}$ ). Hence, we design the ' 0 ' element as a metamaterial particle with a 0 phase response and the ' 1 ' element as a metamaterial particle with a $\pi$ phase response. In this way, the phase responses of the ' 0 ' and ' 1 ' elements are simply defined as $\varphi_{n}=n \pi,(n=0,1)$. The simplest ' 0 ' and ' 1 ' elements can be chosen as perfectly magnetic and electric conductors. However, to reach a broad frequency band, we utilize a subwavelength square metallic
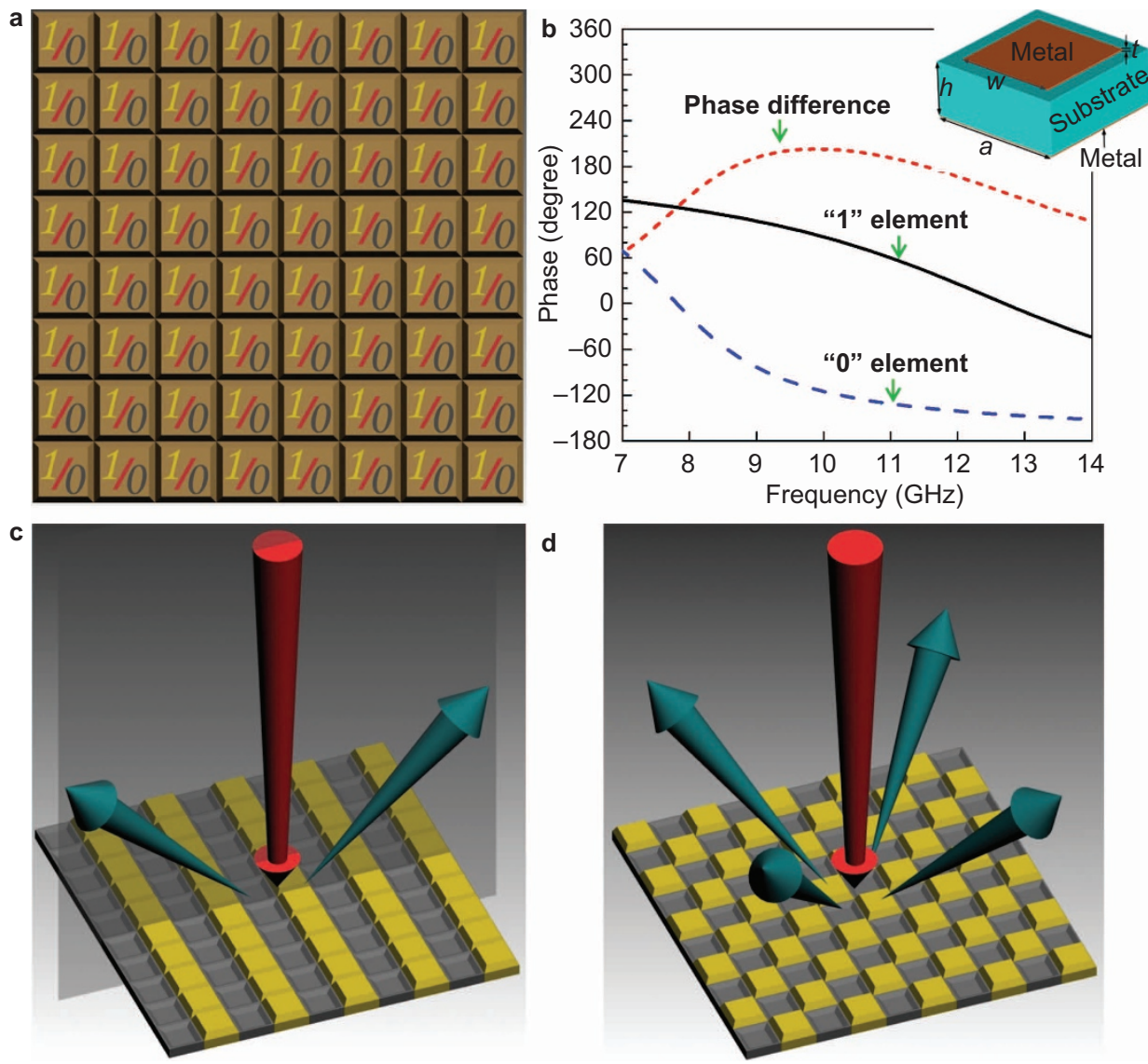

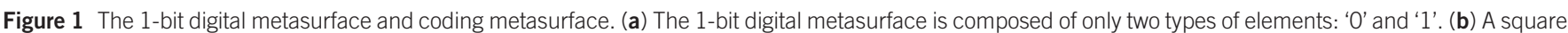

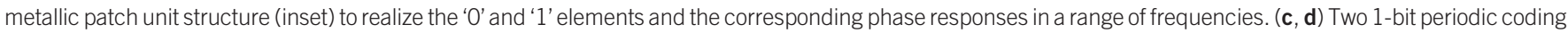

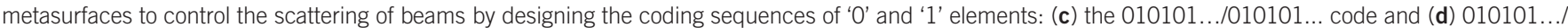
101010... code. 


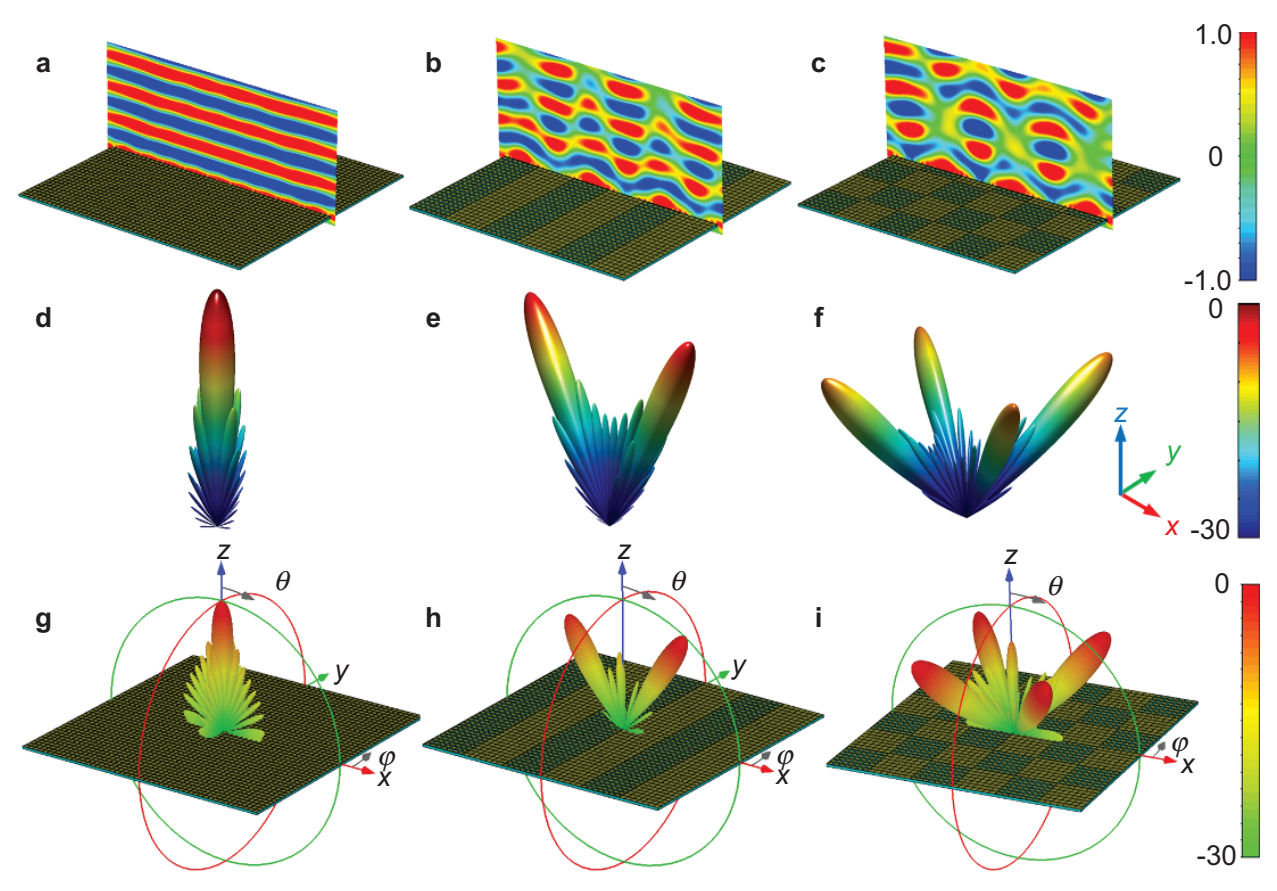

Figure 2 Full-wave simulation results of 1-bit periodic coding metasurfaces to demonstrate their ability to control scattering patterns by different coding sequences under the normal incidence of EM waves. (a-c) The 1-bit metasurface structures with periodic coding sequences: (a) $000000 \ldots / 000000 \ldots$, (b) $010101 \ldots / 010101 \ldots$, (c) $010101 \ldots / 101010 \ldots$ and their corresponding near-field distributions on the observation planes vertical to the metasurfaces. (d-f) The analytical results calculated by Equation (1) of the coding metasurfaces with coding sequences: (d) $000000 \ldots / 000000 \ldots$, (e) $010101 \ldots / 010101 \ldots$ and (f) $010101 \ldots / 101010 \ldots$, from which a single main scattering beam, two main beams, and four main beams are generated by using different coding sequences. (g-i) The full-wave simulation results of coding metasurfaces with the coding sequences of (g) $000000 \ldots / 000000 \ldots$, (h) $010101 \ldots / 010101 \ldots$ and (i) $010101 \ldots / 101010 \ldots$, which are in good agreement with the analytical predictions. EM, electromagnetic.

patch printed on a dielectric substrate to realize the binary elements (see the inset of Figure 1b). The substrate has a thickness of $h=1.964 \mathrm{~mm}$ with a dielectric constant of 2.65 and loss tangent of 0.001 ; the metallic patch has a thickness of $t=0.018 \mathrm{~mm}$ with a width of $w$; and the periodicity of the unit cell is $a=5 \mathrm{~mm}$. When the patch widths are designed as 4.8 and $3.75 \mathrm{~mm}$, the phase difference is approximately $180^{\circ}$ in a broad band. In particular, from $8.1 \mathrm{GHz}$ to $12.7 \mathrm{GHz}$, the phase difference ranges from $135^{\circ}$ to $200^{\circ}$ (it is exactly $180^{\circ}$ at 8.7 and $11.5 \mathrm{GHz}$ ). Hence, we use the patch particle with $w=4.8 \mathrm{~mm}$ as the ' 0 ' element and that with $w=3.75 \mathrm{~mm}$ as the ' 1 ' element, which are easily fabricated in a single-layered dielectric board to construct metasurfaces. Note that the absolute phase response of the ' 0 ' element may not be 0 at a specific frequency, but this case does not affect any physics because the phase can be normalized to 0 .

In contrast to the existing analog metamaterials that utilize effective medium parameters or special dispersion relations to control EM fields, the coding metamaterials simply manipulate EM waves through different coding sequences of ' 0 ' and ' 1 ' elements. For example, under the periodic coding sequence of $010101 \ldots / 010101 \ldots$, the normally incident beam will mainly be reflected to two symmetrically oriented directions by the metasurface, whereas under the periodic coding sequence of $010101 \ldots / 101010 \ldots / 010101 \ldots / 101010 \ldots,{ }^{40}$ the normally incident beam will mainly be reflected to four symmetrically oriented directions, as illustrated in Figure 1c and 1d. To quantitatively illustrate the above physical phenomena, we consider a general square metasurface that contains $N \times N$ equal-sized lattices with dimension $D$ in which each lattice is occupied by a sub-array of ' 0 ' or ' 1 ' elements, as shown in Supplementary Fig. S1. The distribution of ' 0 ' and ' 1 ' lattices can be arbitrary. The scattering phase of each lattice is assumed to be $\varphi(m, n)$, which is either $0^{\circ}$ or $180^{\circ}$. Under the normal incidence of plane waves, the far-field function scattered by the metasurface is expressed as:

$$
\begin{aligned}
& f(\theta, \varphi)=f_{e}(\theta, \varphi) \\
& \sum_{m=1}^{N} \sum_{n=1}^{N} \exp \{-i\{\varphi(m, n)+k D \sin \theta[(m-1 / 2) \cos \varphi+(n-1 / 2) \sin \varphi]\}\}
\end{aligned}
$$

where $\theta$ and $\varphi$ are the elevation and azimuth angles of an arbitrary direction, respectively, and $f_{e}(\theta, \varphi)$ is the pattern function of a lattice. Note that the absolute phase response of the ' 0 ' element can be placed in $f_{e}(\theta, \varphi)$ to ensure that its relative phase is 0 . Hence, the directivity function $\operatorname{Dir}(\theta, \varphi)$ of the metasurface can be given as

$$
\operatorname{Dir}(\theta, \varphi)=4 \pi|f(\theta, \varphi)|^{2} / \int_{0}^{2 \pi} \int_{0}^{\pi / 2}|f(\theta, \varphi)|^{2} \sin \theta \mathrm{d} \theta \mathrm{d} \varphi
$$

Table 1 The optimized codes for different lattice numbers $\boldsymbol{N}$

\begin{tabular}{lcc}
\hline$N$ & Code sequence & RCS reduction $(\mathrm{dB})$ \\
\hline 6 & 001011 & -12.08 \\
7 & 0011010 & -14.64 \\
8 & 00110101 & -15.82 \\
10 & 0001010110 & -18.39 \\
12 & 001001110101 & -19.75 \\
14 & 00111110110101 & -21.41 \\
16 & 0011110110101010 & -22.37 \\
20 & 01000100110000110101 & -23.58 \\
\hline
\end{tabular}

RCS, radar cross-sections. 
a

a
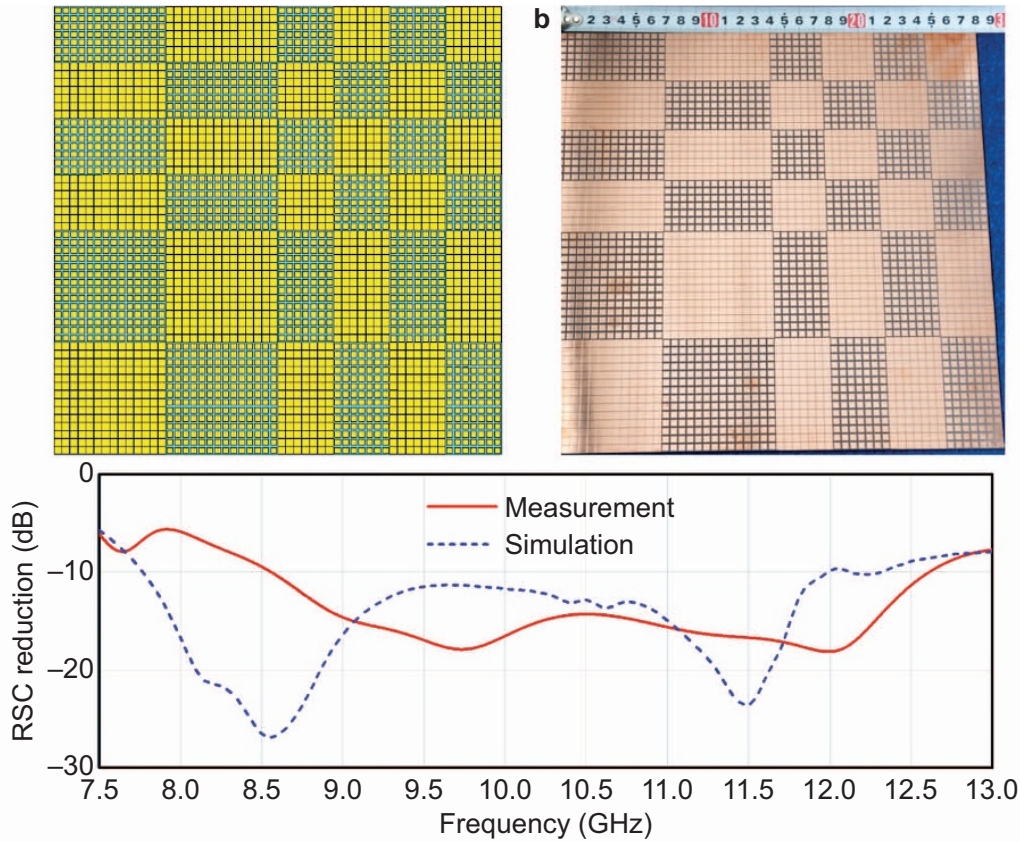

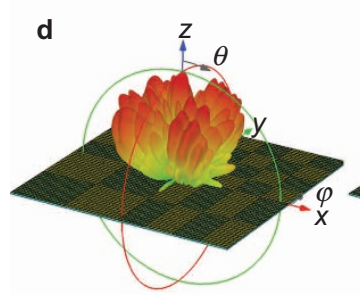

e
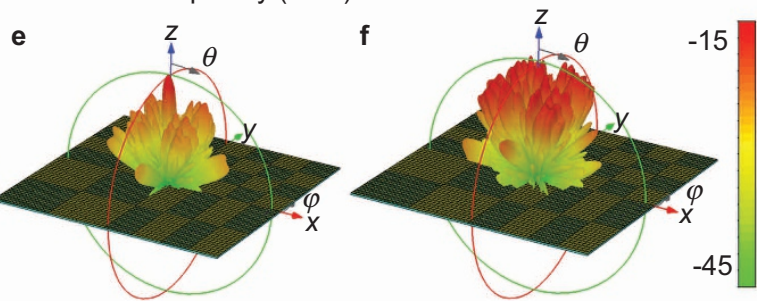

Figure 3 Full-wave simulation and measurement results of a non-periodic 1-bit coding metasurface to suppress the main scattering beams under the normal incidence of EM waves. (a) The metasurface structure with an optimized 1-bit coding sequence given in Table 1. (b) The fabricated sample in an F4B dielectric substrate of the optimized 1-bit coding metasurface. (c) The simulation and measurement results of monostatic RCS reductions over a wide frequency range from 7.5 to $13 \mathrm{GHz}$. (d-f) The simulation results of 3D bistatic RCS patterns at (d) $8 \mathrm{GHz},(\mathbf{e}) 10 \mathrm{GHz}$ and (f) $11.5 \mathrm{GHz}$. It is observed that the coding metasurface redirects the incident EM energy to all directions, and the EM energy in each direction is very small, making significant reductions in both monostatic and bistatic RCSs. 3D, threedimensional; EM, electromagnetic; RCS, radar cross-section.

a
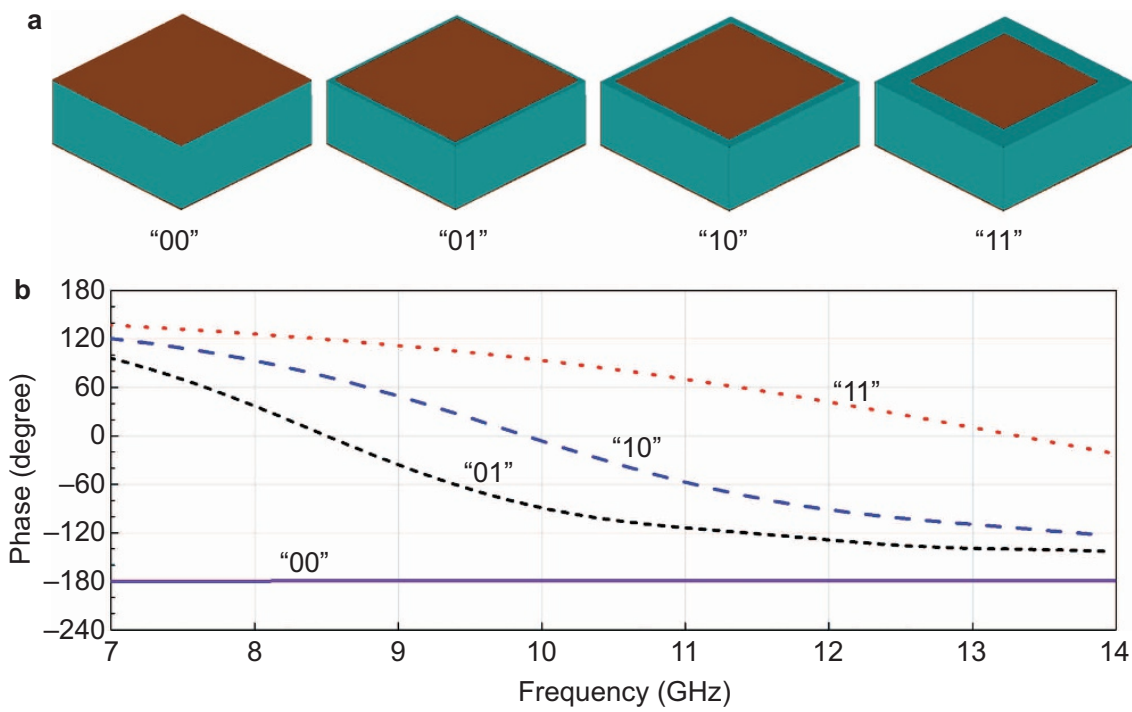

Figure 4 The 2-bit coding metasurface elements and their phase responses. (a) The '00', '01', ' 10 ' and ' 11 ' elements (from left to right) realized by square metallic patches with different sizes. (b) The phase responses of the '00', '01', '10' and ' 11 ' elements. 
Here, the $f_{e}(\theta, \varphi)$ term has been eliminated. From the above equations, we clearly observe control over the scattered fields through coding of the metasurface lattices.

For example, when all lattices are set as ' 0 ' elements, we easily derive that $\left|f_{1}(\theta, \varphi)\right|=C_{1}\left|\cos \psi_{1}+\cos \psi_{2}\right|$; when the coding sequence is chosen as that in Figure 1c or 1d, we have $\left|f_{2}(\theta, \varphi)\right|=C_{2}\left|\sin \psi_{1}+\sin \psi_{2}\right|$ or $\left|f_{3}(\theta, \varphi)\right|=$ $C_{3}\left|\cos \psi_{1}-\cos \psi_{2}\right|$, in which $C_{1}, C_{2}$ and $C_{3}$ are constants, $\psi_{1}=1 / 2 k D$ $(\sin \theta \cos \varphi+\sin \theta \sin \varphi)$ and $\psi_{2}=1 / 2 k D(-\sin \theta \cos \varphi+\sin \theta \cos \varphi)$. The analyses of the above expressions in the Supplementary Information show that the normally incident waves will be scattered as a single main beam, two main beams and four main beams, respectively, using different coding patterns, as confirmed by the full-wave simulations presented in Figure 2. Note that the analytical predictions (Figure $2 \mathrm{~d}-2 \mathrm{f}$ ) are in good agreement with the full-wave simulations (Figure 2g-2i) in all cases. Hence, we can use Equations (1) and (2) to design complicated coding sequences to realize advanced functionalities of coding metasurfaces.

As an application, we aim to reduce the RCSs of metallic surfaces by appropriately coding ' 0 ' and ' 1 ' elements. In fact, the invisibility cloak is one approach to reduce RCSs by forcing EM waves to bend around the target, ${ }^{5,6}$ and the perfect absorber is another approach for reducing RCSs by absorbing all incident EM waves. ${ }^{48,49}$ Here, we propose a new mechanism for reducing the monostatic and bistatic RCSs by redirecting EM energies to all directions through the use of a special ' 0 ' and ' 1 ' coding. Relative to a metallic plate with the same size, the RCS reduction caused by the coding metasurface is obtained as

$$
\operatorname{RCS} \text { reduction }=\frac{\lambda^{2}}{4 \pi N^{2} D^{2}} \underset{\theta, \varphi}{\operatorname{Max}}[\operatorname{Dir}(\theta, \varphi)]
$$

where $\lambda$ is the wavelength in free space. The best RCS reduction can be achieved through optimizing the coding sequences of ' 0 ' and ' 1 ' lattices, and the optimized codes for different numbers of lattices $(N)$ are listed in Table 1 when $D$ is fixed to $\lambda$, in which the code sequences along the horizontal and vertical directions are the same. In fact, the optimized codes can operate in the broadband. Although such codes are obtained at the fixed $D=\lambda$, the RCS reduction remains nearly invariant when $D / \lambda$ changes from 0.6 to 3.0, as shown in Supplementary Fig. S2a.
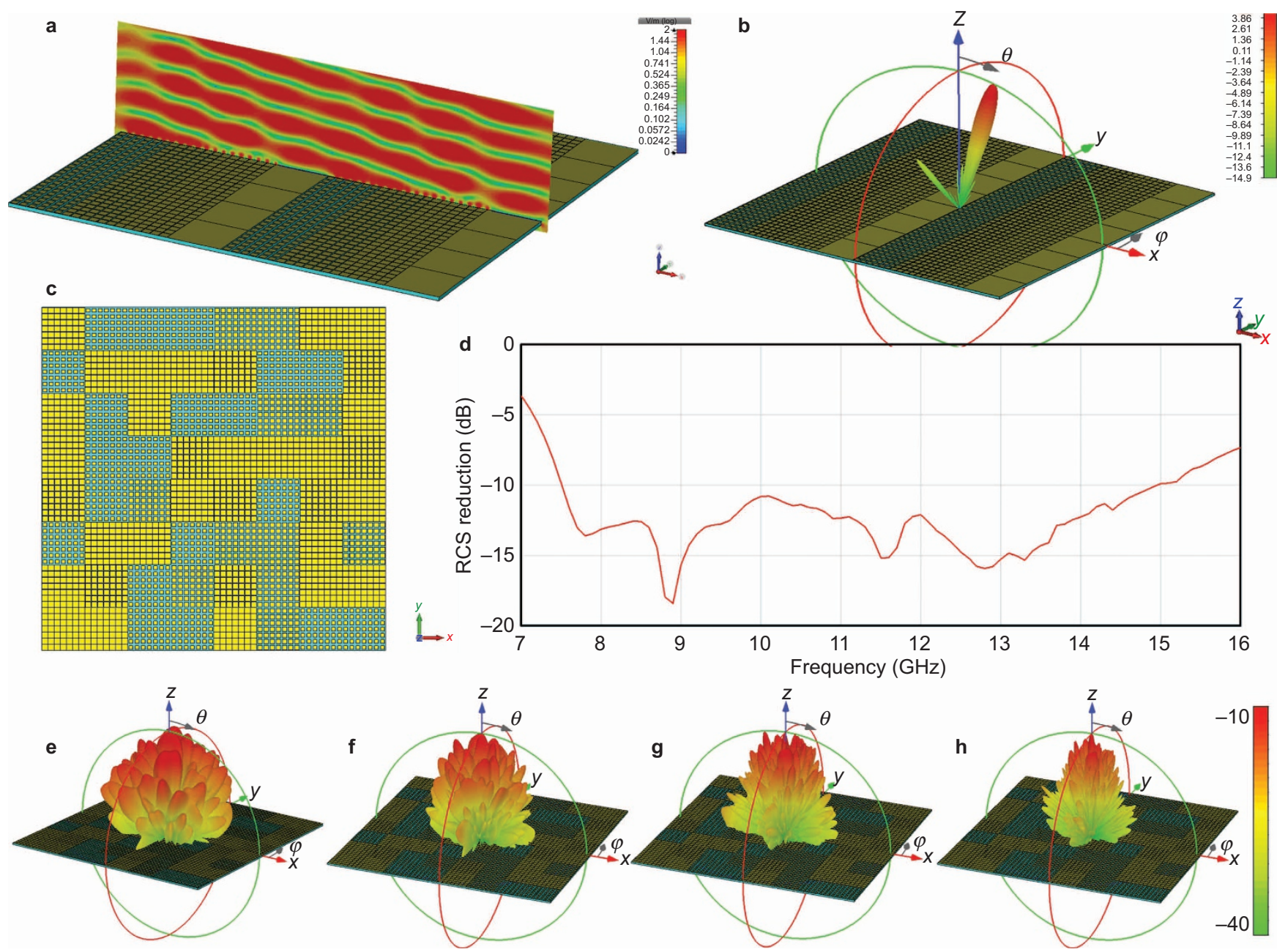

Figure 5 The 2-bit coding metasurfaces and their control over EM waves under different coding sequences. (a) A periodic 2-bit coding metasurface with the coding sequence $0001101100011011 \ldots$ and the near-field distribution on an observation plane vertical to the metasurface. (b) The 3D far-field scattering pattern of the periodic 2-bit coding metasurface. From near-and far-field results, we observe that the normally incident plane waves are reflected to an oblique angle, which is consistent with the generalized Snell's law due to the gradient phase change of the ' 00 ', '01', ' 10 ' and ' 11 ' elements. (c) A non-periodic metasurface constructed using an optimized 2-bit coding sequence. (d) The simulation results of monostatic RCS reductions over a wide frequency range from 7 to $16 \mathrm{GHz}$. (e-h) The simulation results of 3D bistatic RCS patterns at $8 \mathrm{GHz}(\mathbf{e}), 10 \mathrm{GHz}(\mathbf{f}), 13 \mathrm{GHz}(\mathbf{g})$ and $15 \mathrm{GHz}(\mathbf{h})$. The 2-bit coding metasurface can provide a significant RCS reduction over a wider frequency band than the 1-bit coding metasurface. 3D, three-dimensional; EM, electromagnetic; RCS, radar cross-section. 
Note that better RCS reduction is achieved for larger $N$. When $N=20$, the $\mathrm{RCS}$ reduction is as great as $-23 \mathrm{~dB}$ in a wide frequency band. To further guarantee broadband performance, we show that the optimized codes are approximately valid when the phase difference between the ' 0 ' and ' 1 ' elements is in the vicinity of $180^{\circ}$ (Supplementary Fig. S2b). For all cases, when the phase difference varies from $145^{\circ}$ to $215^{\circ}$, a RCS reduction of at least $10 \mathrm{~dB}$ is guaranteed.

To verify the above physical phenomena, we design and fabricate a metasurface based on the optimized coding sequences, as shown in Figure $3 \mathrm{a}$ and $3 \mathrm{~b}$, which contains $8 \times 8$ lattices. The edge length of the metasurface is $280 \mathrm{~mm}$, the width of each lattice is $35 \mathrm{~mm}$ and each lattice is composed of $7 \times 7$ ' 0 ' or ' 1 ' elements. The patterns in Figure $3 \mathrm{a}$ and $3 \mathrm{~b}$ are designed symmetrically from the optimized coding sequence 00110101 for $N=8$ in both the horizontal and vertical directions. During the fabrication, a commercial dielectric board (F4B) is used, which has exactly the same parameters as those in the simulations. The simulation results of monostatic RCS reductions obtained using the commercial software CST Microwave Studio are presented in Figure $3 \mathrm{c}$ for a wide frequency range under the normal incidence (dashed line). Note that the $10-\mathrm{dB}$ bandwidth of the RCS reduction in the backward direction ranges from 7.8 to $12 \mathrm{GHz}$, which is consistent with that for the phase difference. The experimental results (solid line in Figure 3c) obtained from a measurement system, which are shown in Supplementary Fig. S3, confirm the significant reduction of monostatic RCSs in the broadband. To observe bistatic scattering features, threedimensional (3D) scattering patterns of the metasurface are presented in Figure $3 \mathrm{~d}-3 \mathrm{f}$ at three representative frequencies of 8,10 and 11.5 GHz. As shown, the scattered fields are suppressed in low levels in all directions, and the normalized bistatic RCSs are always less than $-10 \mathrm{~dB}$. This is because the coding metasurface has been designed to redirect the incident EM energies to all directions, and in each direction, the energy is small based on the energy conservation principle. At $10 \mathrm{GHz}$, the small peak (below $-10 \mathrm{~dB}$ ) in the direction of the incident wave is caused by the relatively large phase difference (approximately $203^{\circ}$ ) (Figure 1b).

\section{2-bit coding metamaterials}

The concept of the coding metamaterial can be extended from 1-bit coding to 2-bit coding or higher. In 2-bit coding, four types of unit cells with distinct responses are required to mimic the ' 00 ', ' 01 ', ' 10 ' and ' 11 ' elements. The 2-bit coding has greater freedom to manipulate EM waves than 1-bit coding, but requires more complex technology for digital control. Similar to the 1-bit case, the four types of unit cells in 2-bit coding metamaterials should have phase responses of $0, \pi / 2, \pi$ and $3 \pi / 2$, corresponding to the ' 00 ', ' 01 ', ' 10 ' and ' 11 ' elements, respectively. Hence, the phase responses $\varphi_{n}$ are simply defined as $\varphi_{n}=n \pi / 2,(n=0,1,2,3)$.

To realize these metamaterial particles, we still make use of square metallic patches with different sizes printed on a dielectric substrate to design the ' 00 ', ' 01 ', ' 10 ' and ' 11 ' elements in the broadband, as shown in Figure 4. With 2-bit coding metamaterials or metasurfaces, we have greater flexibility in controlling the coding sequences to reach wider applications. For example, we design a simply periodic coding sequence $0001101100011011 \ldots$, as depicted in Figure 5a. Because the adjacent four elements ' 00 ', ' 01 ', ' 10 ' and ' 11 ' have a gradient phase change, based on the generalized Snell's law, ${ }^{32,34}$ the normally incident waves will be reflected at oblique angles, as confirmed by the simulation results of near fields and by the $3 \mathrm{D}$ far-field scattering patterns illustrated in Figure 5a and 5b. As an application, we optimize the 2-bit codes on the same-size metasurface as that in the 1-bit coding to reduce the RCSs of a metallic surface. The optimized 2-bit codes for this purpose are shown in Figure $5 \mathrm{c}$. The simulation results of the monostatic RCSs demonstrate the better performance of the 2-bit coding metasurface (Figure $5 \mathrm{~d}$ ), in which the RCS reduction is below $-10 \mathrm{~dB}$ over a much wider frequency band from 7.5 to $15 \mathrm{GHz}$. The $3 \mathrm{D}$ scattering patterns at $8,10,13$ and $15 \mathrm{GHz}$ shown in Figure $5 \mathrm{e}-5 \mathrm{~h}$ further confirm the strong ability of 2-bit coding metasurface to suppress the bistatic RCSs.

\section{RESULTS AND DISCUSSION}

Based on the concept of coding metamaterials, we are able to not only control EM waves by changing the coding sequences of ' 0 ' and ' 1 ' (or ' 00 ', ' 01 ', ' 10 ' and ' 11 ') elements but also create actual digital metamaterials and programmable metamaterials.

\section{Digital metamaterials}

In the above section, two unit cells are used to realize the ' 0 ' and ' 1 ' elements in the 1-bit coding metasurfaces. To digitally control the ' 0 ' and ' 1 ' responses, we propose a unique metamaterial particle, as shown in Figure 6a. Two planar symmetrical metallic structures (Supplementary Fig. S4) are printed on the top surface of the F4B substrate with a dielectric constant of 2.65 and loss tangent of 0.001 , and the structures are connected by a biased diode. Two metallic via holes are drilled to connect the metamaterial structures with two pieces of ground, which are used to input the biased direct current (DC) voltage. The total size of the particle is $6 \times 6 \times 2 \mathrm{~mm}^{3}$, which is approximately $0.172 \times 0.172 \times 0.057 \lambda^{3}$ at the central frequency. The biased diode can be controlled by DC voltage. When the biased voltage is $3.3 \mathrm{~V}$, the diode is ' $\mathrm{ON}$ ' and the corresponding effective circuit is illustrated in Supplementary Fig. S5a; when there is no biased voltage, the diode is 'OFF', and the corresponding circuit model is given in Supplementary Fig. S5b. The numerical results obtained by inserting the circuit models into CST Microwave Studio demonstrate that the metamaterial particle behaves as a ' 1 ' element when the diode is on and

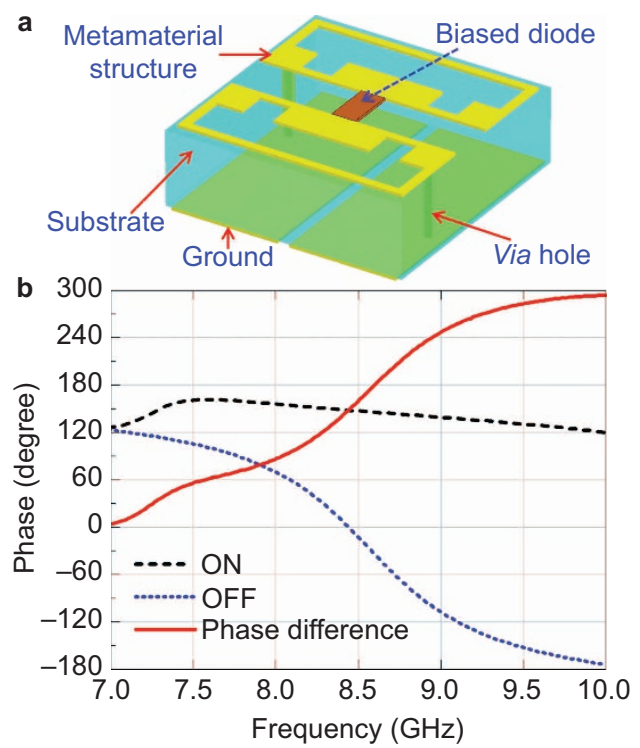

Figure 6 The metamaterial particle for realizing the digital metasurface and the corresponding phase responses. (a) The structure of the metamaterial particle, which behaves as ' $O$ ' and ' 1 ' elements when the biased diode is 'OFF' and 'ON', respectively. (b) The corresponding phase responses of the metamaterial particle as the biased diode is 'OFF' and 'ON' over a range of frequencies. 
as a ' 0 ' element when the diode is off. As clearly shown in Figure 6b, the phase difference is approximately $180^{\circ}$ in the frequency band from 8.3 to $8.9 \mathrm{GHz}$. At $8.6 \mathrm{GHz}$, the phase difference is exactly $180^{\circ}$.

Based on the metamaterial particle, we design and fabricate a sample of a 1-bit digital metasurface, as shown in Supplementary Fig. S6a, $6 \mathrm{c}$ and $6 \mathrm{~d}$. The digital metasurface contains $30 \times 30$ identical unit cells, and each unit cell includes a biased diode (Supplementary Fig. S6d). Every five adjacent columns of the unit cells share a control voltage, which corresponds to a single bit of the control words. Hence, this is a one-dimensional (1D) digital metasurface, and the coding sequence has six control words. As examples, we choose four coding sequences to validate the concepts: $000000,111111,010101$ and 001011 . The simulation results of $3 \mathrm{D}$ scattering patterns from the $1 \mathrm{D}$ digital metasurfaces are presented in Figure $7 \mathrm{a}-7 \mathrm{~d}$, respectively. As shown in Figure $7 \mathrm{a}$ and $7 \mathrm{~b}$, the normally incident beams are directly reflected back with the coding sequences 000000 and 111111 because they mimic perfectly electric and magnetic conductors. Under the periodic coding sequence of 010101 , the normally incident beam is primarily
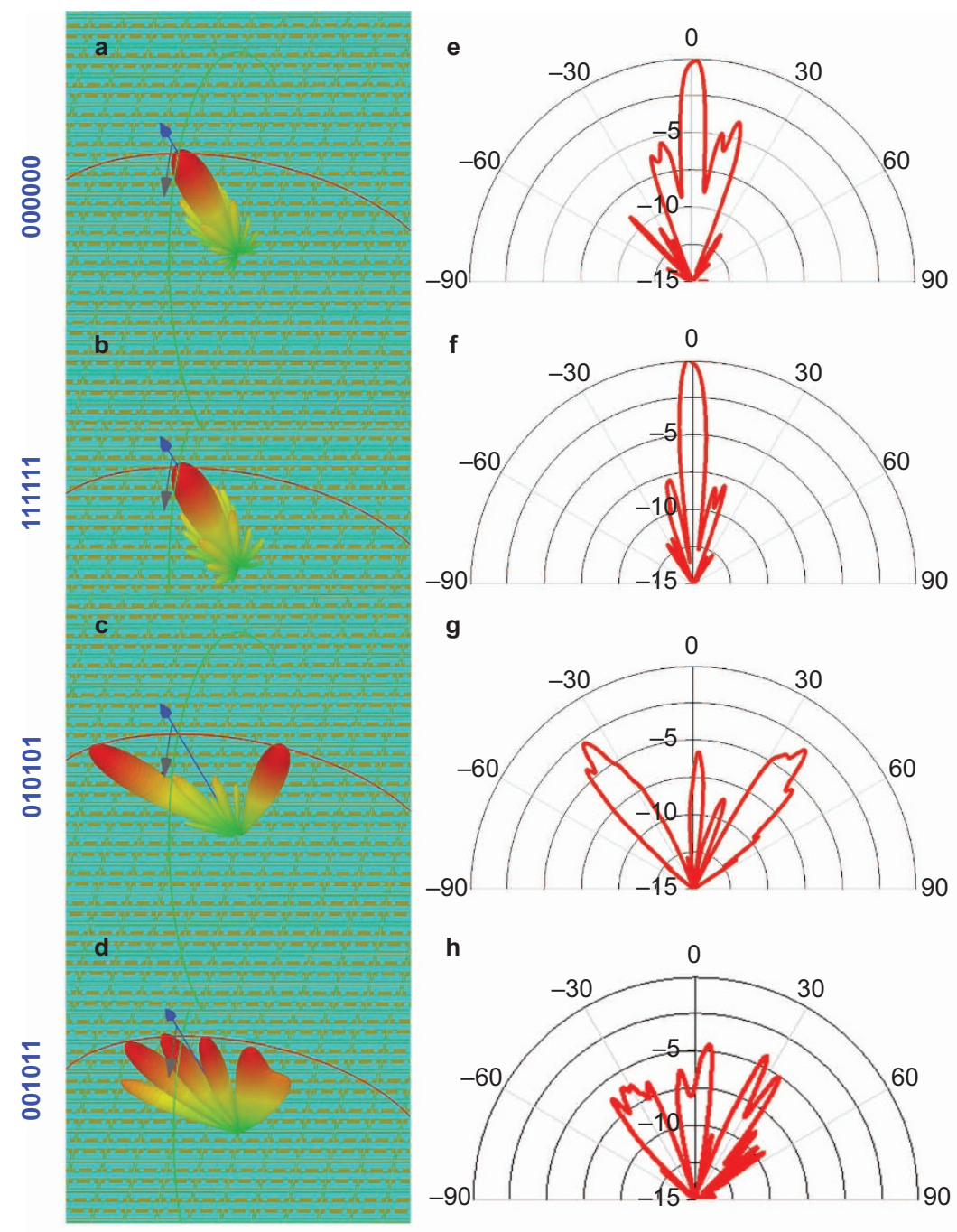

i

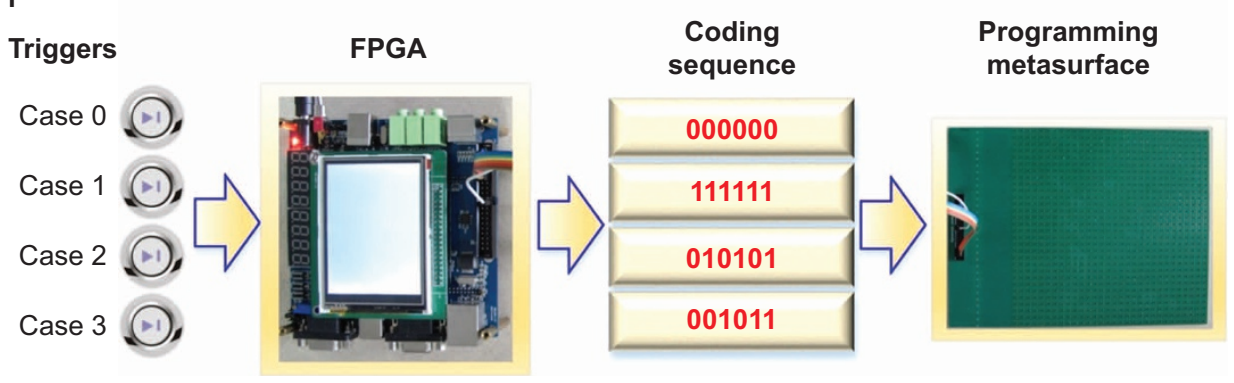

Figure 7 (a-d) Numerical simulation results of scattering patterns at $8.3 \mathrm{GHz}$ for the 1D digital metasurface under different coding sequences: (a) 000000, (b) 111111, (c) 010101 and (d) 001011. (e-f) Experimental results of scattering patterns at $8.6 \mathrm{GHz}$ for the 1D digital metasurface under different coding sequences: (e) 000000, (f) 111111, (g) 010101 and (h) 001011. (i) A flow diagram for realizing a programmable metasurface controlled by the FPGA hardware. 1D, one-dimensional; FPGA, field-programmable gate. 
reflected in two directions by the metasurface, as illustrated in Figure 7c. In the general coding sequence of 001011 , the incident beam is scattered as multiple beams with lower RCS values, as shown in Figure 7d.

\section{Programmable metamaterials}

To digitally control the coding sequences, we design and realize FPGA hardware, ${ }^{50}$ as shown in Supplementary Fig. S6b. Four switches are used as triggers for different controls of coding sequences. When one switch is toggled on, FPGA will output the corresponding coding sequence. Consequently, we can digitally change the voltage distributions on the metasurface by toggling different triggers, which further control the 'ON' and 'OFF' states of the biased diodes, thereby producing the required ' 0 ' and ' 1 ' states of the digital metasurface. Hence, the unique metasurface has different functions controlled by the FPGA program, resulting in a programmable metasurface. A flow chart of the programmable metasurface triggered by FPGA is presented in Figure 7i. Experiments were conducted to verify the multiple abilities of a single metasurface to manipulate EM waves. The measured scattering patterns of the $1 \mathrm{D}$ digital metasurface obtained by triggering coding sequences of $000000,111111,010101$ and 001011 with FPGA are presented in Figure $7 \mathrm{e}-7 \mathrm{~h}$. From these figures, we clearly observe the multiple functionalities, which are in good agreement with the numerical simulations shown in Figure 7a-7d.

\section{CONCLUSIONS}

We have proposed the concepts of coding metamaterials, digital metamaterials and programmable metamaterials and presented designs and realizations of these metamaterials, as well as experiments with these metamaterials. The coding metamaterials are composed of only two types of unit cells, with 0 and $\pi$ phase responses, which we name the ' 0 ' and ' 1 ' elements. By coding ' 0 ' and ' 1 ' elements with controlled sequences (i.e., 1-bit coding), we can manipulate EM waves and realize different functionalities. The concept of the coding metamaterial can be extended from 1-bit coding to 2-bit coding or higher. In 2-bit coding, four types of unit cells, with phase responses of $0, \pi / 2, \pi$ and $3 \pi / 2$, are required to mimic ' 00 ', ' 01 ', ' 10 ' and ' 11 ' elements, respectively. The 2-bit coding metamaterials have greater freedom than 1-bit coding metamaterials to control EM waves.

We also proposed a unique planar metamaterial particle in the subwavelength scale that can realize either the ' 0 ' or ' 1 ' element controlled by a biased diode. Based on this novel metamaterial particle, we designed and realized a digital metamaterial, in which each element has either a ' 0 ' or ' 1 ' state controlled by the biased DC voltage. We have constructed FPGA hardware to control the coding sequence, which is the input for the the digital metamaterial. Hence, the single digital metamaterial can be controlled by the FPGA program to obtain different abilities to manipulate EM waves, thereby realizing a programmable metamaterial.

The proposed coding metamaterials, digital metamaterials and programmable metamaterials are very attractive for a variety of applications, such as controlling the radiation beams of antennas (similarly to phase-array antennas but using a different principle with a considerably less expensive approach), reducing the scattering features of targets and realizing other smart metamaterials. The proposed work can be extended to the millimeter wave and terahertz frequencies.

\section{ACKNOWLEDGEMENTS}

This work was supported in part by the National High Tech (863) Projects (2012AA030402 and 2011AA010202), in part by the National Science Foundation of China $(61138001,60990320$ and 60990324), in part by the 111
Project (111-2-05) and in part by the Joint Research Center on Terahertz Science. TJC greatly appreciates the constructive discussions with N Engheta at the University of Pennsylvania.

1 Cui TJ, Smith DR, Liu R. Metamaterials: Theory, Design, and Applications. New York: Springer Science \& Business Media; 2009.

2 Veselago VG. The electrodynamics of substances with simultaneously negative values of $\varepsilon$ and $\mu$. Soviet Phys Uspekhi 1968; 10: 509-514.

3 Shelby RA, Smith DR, Schultz S. Experimental verification of a negative index of refraction. Science $2001 ; 292$ : 77-79.

4 Pendry JB. Negative refraction makes a perfect lens. Phys Rev Lett 2000; 85: 3966 3969.

5 Pendry JB, Schurig D, Smith DR. Controlling electromagnetic fields. Science 2006; 312: $1780-1782$.

6 Leonhardt U. Optical conformal mapping. Science 2006; 312: 1777-1780.

7 Schurig D, Mock JJ, Justice BJ, Cummer SA, Pendry JB et al. Metamaterial electromagnetic cloak at microwave frequencies. Science 2006; 314: 977-980.

8 Li J, Pendry JB. Hiding under the carpet: a new strategy for cloaking. Phys Rev Lett 2008; 101: 203901.

9 Liu R, Ji C, Mock JJ, Chin JY, Cui TJ et al. Broadband ground-plane cloak. Science 2009; 323: 366-369.

10 Ergin T, Stenger N, Brenner P, Pendry JB, Wegener M. Three-dimensional invisibility cloak at optical wavelengths. Science 2010; 328: 337-339.

11 Ma HF, Cui TJ. Three-dimensional broadband ground-plane cloak made of metamaterials. Nat Commun 2010; 1: 21.

12 Enoch S, Tayeb G, Sabouroux P, Guérin N, Vincent P. A metamaterial for directive emission. Phys Rev Lett 2002; 89: 213902.

13 Silveirinha M, Engheta N. Tunneling of Electromagnetic energy through subwavelength channels and bends using $\varepsilon$-near-zero materials. Phys Rev Lett 2006; 97: 157403.

14 Liu R, Cheng Q, Hand T, Mock JJ, Cui TJ et al. Experimental demonstration of electromagnetic tunneling through an epsilon-near-zero metamaterial at microwave frequencies. Phys Rev Lett 2008; 100: 023903.

15 Zhang B, Luo Y, Liu X, Barbastathis G. Macroscopic invisibility cloak for visible light. Phys Rev Lett 2011; 106: 033901.

16 Chen X, Luo Y, Zhang J, Jiang K, Pendry JB et al. Macroscopic invisibility cloaking of visible light. Nat Commun 2011; 2: 176.

17 Cheng $Q$, Jiang WX, Cui TJ. Spatial power combination for omnidirectional radiation via anisotropic metamaterials. Phys Rev Lett 2012; 108: 213903.

18 Blanco A, Chomski E, Grabtchak S, Ibisate M, John S et al. Large-scale synthesis of a silicon photonic crystal with a complete three-dimensional bandgap near 1.5 micrometres. Nature 2000; 405: 437-440.

19 Sakoda K. Optical Properties of Photonic Crystals. New York: Springer Science \& Business Media; 2005.

20 Jiang WX, Cui TJ, Cheng Q, Chin JY, Yang XM et al. Design of arbitrarily shaped concentrators based on conformally optical transformation of nonuniform rational B-spline surfaces. Appl Phys Lett 2008; 92: 264101.

21 Lai Y, Ng J, Chen H, Han D, Xiao J et al. Illusion optics: the optical transformation of an object into another object. Phys Rev Lett 2009; 102: 253902.

22 Jiang WX, Cui TJ, Yang XM, Ma HF, Cheng Q. Shrinking an arbitrary object as one desires using metamaterials. Appl Phys Lett 2011; 98: 204101.

23 Kundtz N, Smith DR. Extreme-angle broadband metamaterial lens. Nat Mater 2010; 9: 129-132.

24 Ma HF, Cui TJ. Three-dimensional broadband ground-plane cloak made of metamaterials. Nat Commun 2010; 1: 21.

25 Smith DR, Mock JJ, Starr AF, Schurig D. Gradient index metamaterials. Phys Rev E 2005; 71: 036609.

26 Hao Y, Mittra R. FDTD Modeling of Metamaterials: Theory and Applications. Boston: Artech House; 2009.

27 Chen X, Ma HF, Zou XY, Jiang WX, Cui TJ. Three-dimensional broadband and highdirectivity lens antenna made of metamaterials. J Appl Phys 2011; 110: 044904.

28 Lier E, Werner DH, Scarborough CP, Wu Q, Bossard JA. An octave-bandwidth negligible-loss radiofrequency metamaterial. Nat Mater 2011; 10: 216-222.

29 Jiang WX, Qiu CW, Han TC, Cheng Q, Ma HF et al. Broadband all-dielectric magnifying lens for far-field high-resolution imaging. Adv Mater 2013; 25: 6963-6968.

30 Yang XM, Zhou XY, Cheng Q, Ma HF, Cui TJ. Diffuse reflections by randomly gradient index metamaterials. Opt Lett 2010; 35: 808-810.

31 Silva A, Monticone F, Castaldi G, Galdi V, Alù A et al. Performing mathematical operations with metamaterials. Science 2014; 343: 160-163.

32 Yu N, Genevet P, Kats MA, Aieta F, Tetienne JP et al. Light propagation with phase discontinuities: generalized laws of reflection and refraction. Science $2011 ; 334$ 333-337.

33 Ni X, Emani NK, Kildishev AV, Boltasseva A, Shalaev VM. Broadband light bending with plasmonic nanoantennas. Science 2012; 335: 427

34 Sun $\mathrm{S}, \mathrm{He}$ Q, Xiao S, Xu Q, Li X et al. Gradient-index meta-surfaces as a bridge linking propagating waves and surface waves. Nat Mater 2012; 11: 426-431.

35 Yin X, Ye Z, Rho J, Wang Y, Zhang X. Photonic spin hall effect at metasurfaces. Science 2013; 339: 1405-1407.

36 Lin J, Mueller JP, Wang Q, Yuan G, Antoniou N et al. Polarization-controlled tunable directional coupling of surface plasmon polaritons. Science 2013; 340: 331-334. 
37 Miroshnichenko AE, Kivshar YS. Polarization traffic control for surface plasmons. Science 2013; 340: 283-284.

38 Grady NK, Heyes JE, Chowdhury DR, Zeng Y, Reiten MT et al. Terahertz metamaterials for linear polarization conversion and anomalous refraction. Science 2013; 340 : 1304-1307.

39 Della Giovampaola C, Engheta N. Digital metamaterials. Cond Matter Mater Sci2014; ArXiv: 1403.6488. Available at http://arxiv.org/abs/1403.6488 (accessed 23 August 2014).

40 Paquay M, Iriarte JC, Ederra I, Gonzalo R, de Maagt P. Thin AMC structure for radar cross-section reduction. IEEE Trans Antennas Propag 2007; 55: 3630-3638.

41 Mait JN. Design of binary-phase and multiphase Fourier gratings for array generation. J Opt Soc Am A 1990; 7: 1514-1528.

42 Wang MR, Su H. Laser direct-write gray-level mask and one-step etching for diffractive microlens fabrication. Appl Opt 1998; 37: 7568-7576.

43 Coomber SD, Cameron CD, Hughes JR, Sheerin DT, Slinger CW et al. Optically addressed spatial light modulators for replaying computer-generated holograms. Proc SPIE 2001; 4457: 9-19.

44 Shrekenhamer D, Montoya J, Krishna S, Padilla WJ. Four-color metamaterial absorber THz spatial light modulator. Adv Opt Mater 2013; 1: 905-909.

45 Savo S, Shrekenhamer D, Padilla WJ. Liquid crystal metamaterial absorber spatial light modulator for THz applications. Adv Opt Mater 2014; 2: 275-279.
46 Chan WL, Chen HT, Taylor AJ, Brener I, Cich MJ et al. A spatial light modulator for terahertz beams. Appl Phys Lett 2009; 94: 213511.

47 Karl N, Reichel K, Chen HT, Taylor AJ, Brener I et al. An electrically driven terahertz metamaterial diffractive modulator with more than $20 \mathrm{~dB}$ of dynamic range. Appl Phys Lett 2014; 104: 091115.

48 Landy NI, Sajuyigbe S, Mock JJ, Smith DR, Padilla WJ. Perfect metamaterial absorber. Phys Rev Lett 2008; 100: 207402.

49 Chen HT, Zhou J, O'Hara JF, Chen F, Azad AK et al. Antireflection coating using metamaterials and identification of its mechanism. Phys Rev Lett 2010; 105: 073901.

50 Maxfield C. The Design Warrior's Guide to FPGAs: Devices, Tools and Flows. Oxford: Elsevier; 2004.

\section{(c) (i) (2) This work is licensed under a Creative Commons Attribution-}

NC SA NonCommercial-ShareAlike 3.0 Unported License. The images or other third party material in this article are included in the article's Creative Commons license, unless indicated otherwise in the credit line; if the material is not included under the Creative

Commons license, users will need to obtain permission from the license holder to reproduce the material. To view a copy of this license, visit http://creativecommons.org/licenses/by-nc-sa/3.0/

Supplementary information for this article can be found on the Light: Science \& Applications' website (http://www.nature.com/lsa/). 